Document downloaded from:

http://hdl.handle.net/10251/82487

This paper must be cited as:

Sala, A.; Pitarch Catalá, JL. (2016). Optimisation of transient and ultimate inescapable sets with polynomial boundaries for nonlinear systems. Automatica. 73:82-87. doi:10.1016/j.automatica.2016.06.017.

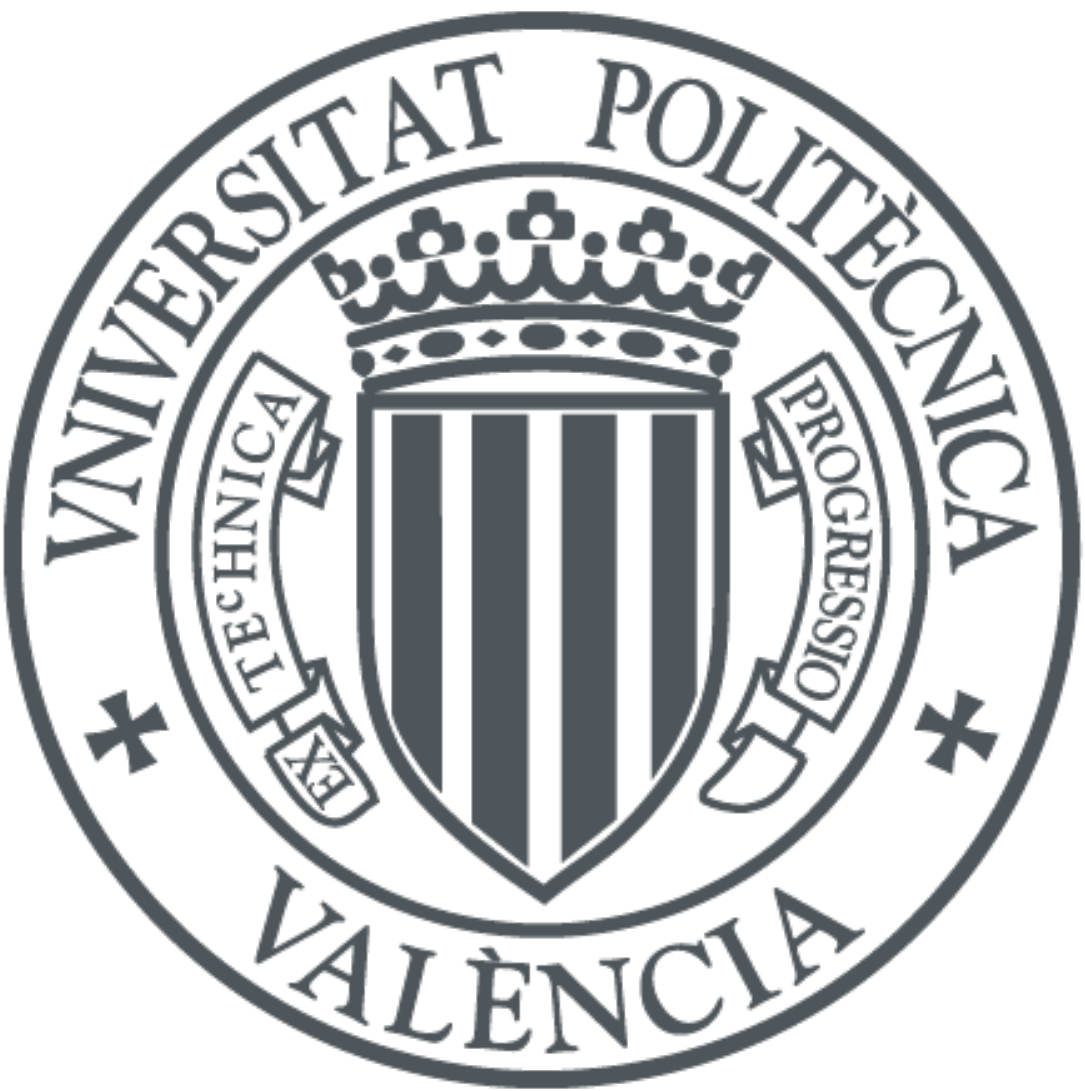

The final publication is available at

http://doi.org/10.1016/j.automatica.2016.06.017

Copyright Elsevier

Additional Information 


\title{
Optimisation of transient and ultimate inescapable sets with polynomial boundaries for nonlinear systems
}

\author{
A. Sala ${ }^{a}$, J.L. Pitarch ${ }^{\mathrm{b}, 1}$ \\ ${ }^{a}$ Instituto Univ. de Automática e Informática Industrial (AI2), Universitat Politècnica de València \\ Camino de Vera S/N, PC:46022, Valencia, Spain. \\ ${ }^{\mathrm{b}}$ Systems Engineering and Control DPT, Escuela de Ingenierías Industriales (EII), Universidad de Valladolid \\ C/ Real de Burgos S/N, PC:47011, Valladolid, Spain.
}

\begin{abstract}
This paper addresses the problem of bounding the trajectories of nonlinear systems (transient and ultimate bounds) from initial conditions in given sets, when subject to possibly nonvanishing disturbances constrained by some finite-interval integral bounds, with a suitable controller. The so-called robustly-inescapable sets are determined from such initial conditions and disturbance bounds. In order to get numerical results, the approach considers embedding the nonlinear dynamics in a convex combination of polynomials, and solving sum-of-squares (SOS) problems on them, optimising some inescapable-set size parameters. Determination of approximate (locally) optimal solutions usually requires an iterative evaluation of SOS problems, because of products of decision variables.
\end{abstract}

Key words: disturbance rejection; inescapable set; reachability; persistent disturbance; SOS tools; polynomial methods

\section{Introduction}

Domain of attraction (DA) estimates for undisturbed nonlinear dynamic systems can be obtained from Lyapunov level sets [7]. In order to get a systematic numerical procedure, smooth nonlinear systems can be embedded onto a parameter varying dynamics (linear -LPV-, or polynomial -PPV-) via quasi-LPV modelling [20] or Taylor-series approaches $[17,6]$, valid in a local region. With these models and semidefinite programming, conservative estimates of the DA can be obtained $[5,7,10,16]$.

In disturbed systems, domain of attraction analysis must be transformed to: $(a)$ bounding some integral norm of the state or $(b)$ determining inescapable (a.k.a. disturbance-invariant) sets $[3,11]$.

In case $(a)$, well-known literature uses Linear Matrix Inequality (LMI) conditions in order to guarantee a bound on the induced $\mathcal{L}_{2} \mapsto \mathcal{L}_{2}$ norm [8], the energy-to-peak $\mathcal{L}_{2} \mapsto \mathcal{L}_{\infty}[15]$, or the peak-to-peak $\mathcal{L}_{\infty} \mapsto \mathcal{L}_{\infty}$ case [1].

In case $(b)$, inescapable sets are strongly related to

Email addresses: asala@isa.upv.es (A. Sala), jose.pitarch@autom.uva.es (J.L. Pitarch).

1 Corresponding author. peak-to-peak bounding. However, the optimal controller minimizing the $\mathcal{L}_{1}$ norm (induced peak to peak) of continuous-time linear systems cannot be expressed in terms of LMIs (in [1], the $\star$-norm is minimized as a bound for it). A straightforward extension of [1] to polytopic systems appears in, for instance, [18], but there are no significant alternative proposals in nonlinear/polynomial literature to the authors' knowledge. The concept of inescapable set is refined considering transient and ultimate bounds in [9], being a solution provided for the linear switching case. There are other approaches to set invariance, such as probabilistic ones [14], or transient bounds in adaptive control [2], out of the scope of this paper.

In quasi-LPV/PPV cases, precise evaluation of inescapable sets is of key importance: as such models are, in general, only locally valid, it must be ensured that the trajectories do not exit the model-validity region, say, $\Omega$. Determining the set of initial conditions such that $\Omega$ is inescapable from them (and, eventually, some closed-loop performance is attained) is the motivation of this work. The concrete objectives of this paper are:

(1) Considering a class of non-vanishing disturbances characterized by known bounds $\beta(t, h)$ on the integral of some functions over the interval $[t-h, t]$, 
being 2-norm, peak bounds and static uncertainty particular cases.

(2) Giving sufficient conditions to guarantee some transient and ultimate inescapable sets from a given initial condition set, as well as guaranteeing that certain set is reached in finite time (guaranteed visit).

(3) Proposing optimisation setups on the above problems for some relevant cases.

The structure of the paper is as follows: next section discusses preliminary concepts and problem statement, Section 3 presents a sufficient Lyapunov-like inescapability inequality, Section 4 casts the problem for PPV systems as SOS conditions, an academic example is shown in Section 5 and a conclusions section closes the paper.

\section{Notation and problem statement}

Consider a nonlinear continuous-time system

$$
\dot{x}(t)=f(x(t))+B(x(t)) u(t)+e(x(t), w(t))
$$

where $x(t) \in \mathbb{R}^{n}$ is the state vector, $u(t) \in \mathbb{R}^{c}$ is the control input, $w(t) \in \mathbb{R}^{d}$ is an external disturbance vector and $f(\cdot), B(\cdot), e(\cdot, \cdot)$ are smooth functions of the state with appropriate dimensions. State-feedback control $u(t):=u(x(t))$ will be considered in the sequel.

Disturbances $w(\cdot)$ and state will be assumed to fulfill some integral constraints, stated a priori:

$$
\begin{aligned}
& \int_{0}^{h} L_{k}(w(t-\tau), x(t-\tau)) d \tau \leq \beta^{[k]}(t, h) \\
& \forall t \geq h \geq 0, \forall k=1, \ldots, S
\end{aligned}
$$

where $\beta^{[k]}(t, h)$ is known, $\beta^{[k]}(t, 0)=0, \beta_{h}^{[k]}(t, h):=$ $\frac{\partial \beta^{[k]}}{\partial h}$ is continuous and nonnegative in $0 \leq h \leq t$, and $L_{k}(\cdot, \cdot)$ are known continuous functions. Shorthand $L_{k}(t-\tau)$ will be used instead of $L_{k}(w(t-\tau), x(t-\tau))$. Time-invariant bounds will be shorthanded omitting the dependence of $\beta^{[k]}$ on $t ; k$ will be omitted when $S=1$.

The triplets of functions of time $(x(t), u(t), w(t))$, for $t \geq 0$, compatible with the model (1) and constraints (2) will be denoted as $\mathcal{G}$. The constraints imply, when $h \rightarrow 0$, instantaneous constraints $L_{k}(w(t), x(t)) \leq \beta_{h}^{[k]}(t, 0)$, motivating definition of $\mathcal{W}(x):=\left\{w: L_{k}(w, x) \leq\right.$ $\left.\sup _{t \geq 0} \beta_{h}^{[k]}(t, 0)\right\}$ for later use.

Let us denote, if suitable limits exist:

$$
\bar{\beta}^{[k]}(t, h):=h^{-1} \beta^{[k]}(t, h), \quad \bar{\beta}_{\infty}^{[k]}:=\liminf _{t \rightarrow \infty} \bar{\beta}^{[k]}(t, t)
$$

Constraints (2) can be used, e.g., to assert disturbance power constraints with $L=w^{T} w: \bar{\beta}(t, h)^{1 / 2}$ is a bound for the root mean square (RMS) average value in an interval of $h$ time units; $\bar{\beta}(t, t)^{1 / 2}$ denotes the maximum
RMS value of $w$ prior to time $t$; if $\bar{\beta}_{\infty} \geq \epsilon$, non-vanishing disturbances are admissible; $\beta(t, h)=\gamma h$ would model peak bounds $\|w(t)\|^{2} \leq \gamma$; setting $\beta_{h}(t, h)=\gamma(t-h)$ would model $\|w(t)\|^{2} \leq \gamma(t)$. If $\beta_{h}^{[k]}$ is high, the bounds approximate those of impulsive disturbances in a limit case. As a last example, a set of finite-time power bounds $\int_{0}^{T_{i}} w^{t} w \leq \gamma_{i}$, such that $0=T_{0}<T_{1}<\cdots<T_{n}, \gamma_{1}<$ $\cdots<\gamma_{n}$ can be asserted with $\beta(t, h)=\kappa \cdot \gamma_{n}+\gamma_{i}$ for $\kappa$ and $i$ such that $\kappa \cdot T_{n}+T_{i-1} \leq h \leq \kappa \cdot T_{n}+T_{i}$.

Other cases, such as $L_{k}=w^{T} M_{w} w-\gamma x^{T} M_{x} x, \beta^{[k]}=0$ can be used to take system uncertainty into account.

Given $\mathcal{G}$ and an initial-condition set $\Theta_{i}$, the following definitions will be used throughout the paper:

Definition $1 A$ set $\Theta_{T}$ is inescapable from $\Theta_{i}$ with a controller $u(x)$ if

$$
\begin{aligned}
(x(\cdot), u(x(\cdot)), w(\cdot)) \in \mathcal{G} \quad \text { and } & x(0) \in \Theta_{i} \\
\Rightarrow & x(t) \in \Theta_{T} \forall t \geq 0
\end{aligned}
$$

Definition 2 A set $\Theta_{\infty}$ is ultimately inescapable from $\Theta_{i}$ with a controller $u(x)$ if there exists $t_{U} \geq 0$ such that

$$
\begin{aligned}
(x(\cdot), u(x(\cdot)), w(\cdot)) \in \mathcal{G} & \text { and } \quad x(0) \in \Theta_{i} \\
& \Rightarrow x(t) \in \Theta_{\infty} \forall t \geq t_{U}
\end{aligned}
$$

Definition 3 A set $\Theta_{v} \subset \Omega$ is a guaranteed-visit set from $\Theta_{i}$ with a controller $u(x)$ if:

$$
\begin{aligned}
& (x(\cdot), u(x(\cdot)), w(\cdot)) \in \mathcal{G} \quad \text { and } \quad x(0) \in \Theta_{i} \\
& \Rightarrow \exists t_{v} \geq 0 \text { such that } x\left(t_{v}\right) \in \Theta_{v}
\end{aligned}
$$

By definition, an inescapable set is ultimately inescapable, and both are, too, guaranteed-visit ones.

Problem statement: The goal of this paper is setting up sufficient conditions to determine sets fulfilling the above definitions for model (1) and constraints (2), generalising the related results cited in the introduction.

Defining semialgebraic sets with polynomial boundaries (defined by strict - open sets- or non-strict -closed sets-inequalities) and polynomial $L_{k}(w, x), f(x), B(x)$, $e(x, w), u(x)$ (or convex combination thereof) will allow posing sufficient conditions as sum-of-squares problems for which software tools exist allowing to obtain numerical solutions; these issues will be discussed in section 4 .

\section{$3 \quad$ Inescapability conditions}

Theorem 1 If there exist an open region $\Omega \subset \mathbb{R}^{n}$, a controller $u(x)$, a nonnegative function $V(x)$ and scalars $\alpha \geq 0, \lambda_{k} \geq 0, k=1, \ldots, S$, fulfilling

$$
\dot{V}(x(t))+\alpha V(x(t))-\sum_{k=1}^{S} \lambda_{k} L_{k}(w(t), x(t)) \leq 0
$$


for all $x(t) \in \Omega, w(t) \in \mathcal{W}(x(t))$, then, the time evolution of $V(x(t))$ can be bounded by

$$
\begin{aligned}
V(x(t)) & \leq \psi\left(V_{0}, \alpha, \lambda, t\right) \\
& :=e^{-\alpha t} V_{0}+\sum_{k=1}^{S} \lambda_{k} \int_{0}^{t} \beta_{h}^{[k]}(t, \tau) e^{-\alpha \tau} d \tau
\end{aligned}
$$

for every trajectory $(x(t), u(x(t)), w(t)) \in \mathcal{G}$ if the closed set $\Theta(\tau):=\left\{x: V(x) \leq \psi\left(V_{0}, \alpha, \lambda, \tau\right)\right\}$ fulfills $\Theta(\tau) \subset \Omega$ for all $0 \leq \tau \leq t$, and $x(0) \in \Theta(0)$; actually, $\Theta(0)=\{x$ : $\left.V(x) \leq V_{0}\right\}$.

Thus, defining $\psi_{\max }\left(V_{0}, \alpha, \lambda\right):=\sup _{\tau \geq 0} \psi\left(V_{0}, \alpha, \lambda, \tau\right)$, the set $\Theta_{T}:=\left\{x: V(x) \leq \psi_{\max }\left(\bar{V}_{0}, \alpha, \lambda\right)\right\}$, is inescapable from $\Theta_{i}$ with controller $u(x)$ if

$$
\Theta_{i} \subset \Theta(0) \text { and } \Theta_{T} \subset \Omega
$$

Proof: Consider the first-order differential equation:

$$
\dot{V}(t)+\alpha V(t)=\sum_{k=1}^{S} \lambda_{k} L_{k}(w(t), x(t))-g(t)
$$

where $g(t) \geq 0$ for all $t$, does exist by (7). The solution of (10) is the convolution formula:

$V(t)=e^{-\alpha t} V(x(0))+\int_{0}^{t}\left(\sum_{k=1}^{S} \lambda_{k} L_{k}(t-\tau)-g(t-\tau)\right) e^{-\alpha \cdot \tau} d \tau$

As both $g$ and $e^{-\alpha \tau}$ are nonnegative, if $V(x(0)) \leq V_{0}$ :

$$
V(t) \leq e^{-\alpha t} V_{0}+\sum_{k=1}^{S} \lambda_{k} \int_{0}^{t} L_{k}(t-\tau) e^{-\alpha \cdot \tau} d \tau
$$

Considering $H(t, h):=\int_{0}^{h} L_{k}(t-\tau) d \tau$, as $H(t, 0)=0$, integration by parts formulae (applied twice) gets:

$$
\begin{gathered}
\int_{0}^{t} L_{k}(t-\tau) e^{-\alpha \tau} d \tau=H(t, t) e^{-\alpha t}+\alpha \int_{0}^{t} H(t, \tau) e^{-\alpha \tau} d \tau \\
\leq \beta^{[k]}(t, t) e^{-\alpha t}+\alpha \int_{0}^{t} \beta^{[k]}(t, \tau) e^{-\alpha \tau} d \tau \\
=\int_{0}^{t} \beta_{h}^{[k]}(t, \tau) e^{-\alpha \tau} d \tau
\end{gathered}
$$

so, carrying out the above for each $k,(11)$ can be used to get (8). The condition $\Theta(\tau) \subset \Omega$ for $\tau \leq t$ ensures that the trajectories do not exit the open region where (7), and consequently (8), were valid. As $\Theta(\tau) \subset \Theta_{T}$ for all $\tau \geq 0$, (9) ensures that $\Theta(\tau) \subset \Omega$ for all $\tau \geq 0$. Thus, (8) holds for all $t \geq 0$ and, hence, $\Theta_{T}$ is inescapable.

Corollary 1 If conditions in the Theorem 1 hold, then (a) if $\alpha>0$, the set $\Theta_{\infty}:=\{x: V(x) \leq \vartheta\} \cap \Theta_{T}$, for any $\vartheta>\psi_{\infty}(\alpha, \lambda)$, being $\psi_{\infty}(\alpha, \lambda):=\lim \sup \psi\left(V_{0}, \alpha, \lambda, t\right)$, is ultimately inescapable from $\Theta_{i}$; $\stackrel{t \rightarrow \infty}{(b)}$ If $V_{0}=0$ and $\beta^{[k]}\left(t_{1}, h\right)=\beta^{[k]}\left(t_{2}, h\right)$ for all $t_{1}, t_{2}$, for all $k$, then $\Theta_{T}=\Theta_{\infty}$.
Proof: (a) As $\alpha>0, e^{-\alpha t}$ tends to zero for large $t$, so

$$
\limsup _{t \rightarrow \infty} \psi\left(V_{0}, \alpha, \lambda, t\right)=\psi_{\infty}(\alpha, \lambda) \leq \psi_{\max }\left(V_{0}, \alpha, \lambda\right)
$$

Hence, for any $\vartheta>\psi_{\infty}(\alpha, \lambda)$ there exists $t_{U}$ such that $V(x(t)) \leq \vartheta$ for all $t \geq t_{U}$. Inescapability of $\Theta_{T}$ ensures, too, that $V(x(t)) \leq \psi_{\max }\left(V_{0}, \alpha, \lambda\right) \forall t \geq t_{U}$. Now, $\Theta_{\infty}$ in the Corollary statement is the level set $\{x: V(x) \leq$ $\left.\min \left(\vartheta, \psi_{\max }\left(V_{0}, \alpha, \lambda\right)\right)\right\}$.

(b) As $\beta_{h}^{[k]} \geq 0$, assuming $V_{0}=0$ and $\beta^{[k]}$ being independent of $t$ entails that the bound $\psi(0, \alpha, \lambda, t)$ increases monotonically with $t$, so $\psi_{\max }(0, \alpha, \lambda)=\psi_{\infty}(\alpha, \lambda)$.

Assertion (b) entails that, with time-invariant constraints, "inescapable" and "ultimately inescapable" sets proven by Theorem 1 coincide if $V_{0}=0$.

Particular cases. The bounds for $V(t)$ from the above theorem generalise some particular cases in literature. Indeed, infinite integral bound $\int_{0}^{\infty} w^{T} w \leq \gamma$ ends up in $V(t) \leq e^{-\alpha t}\left(V_{0}+\gamma\right)$, appearing in, for instance, [8], and, for $\alpha>0$, peak bound $\beta(t, h)=\gamma h$ yields

$$
V(t) \leq e^{-\alpha t} V_{0}+\frac{\gamma}{\alpha}\left(1-e^{-\alpha t}\right)
$$

discussed in [1]. Component-wise disturbance bounds [4] can also be trivially considered.

Note: With $\alpha>0$, there might exist $V_{0} \neq 0$ achieving the same $\Theta_{T}$ than the one resulting with $V_{0}=0$, for any $V_{0}$ such that $\psi_{\max }\left(V_{0}, \alpha, \lambda\right)=\psi_{\max }(0, \alpha, \lambda)$. For instance, with peak-bounded disturbances we have, from (12), that any $V_{0} \leq \gamma / \alpha$ ensures $\Theta_{T}=\{V(x) \leq \gamma / \alpha\}$.

Assumption 1 On the sequel, $\psi_{\max }$ and $\psi_{\infty}$ will be assumed to be known ${ }^{2}$ functions, as they can be determined from $\beta$.

Lemma 1 If $\bar{\beta}_{\infty}^{[k]}$ is finite for all $k$, and there exist $\Omega$, $u(x), V(x), V_{0}, \alpha>0, \lambda_{k}$ proving that $\Theta_{T}$ is inescapable from some $\Theta_{i}$ by Theorem 1, with finite $\psi_{\max }\left(V_{0}, \alpha, \lambda\right)$, then, for any $\varepsilon>0$, the set

$$
\Theta_{v}:=\left\{x: V(x) \leq \psi_{v}(\alpha, \lambda)+\varepsilon\right\}
$$

being $\psi_{v}(\alpha, \lambda):=\alpha^{-1} \sum_{k} \lambda_{k} \bar{\beta}_{\infty}^{[k]}$, is a guaranteed-visit set from $\Theta_{i}$ with controller $u(x)$.

Proof: From (11), $V(x(t)) \leq \eta(t)$, being $\eta$ the time response of the first-order linear system, initial conditions and input below:

$$
\dot{\eta}(t)+\alpha \eta(t)=\nu(t) ; \quad \eta(0)=V_{0}, \quad \nu(t)=\sum_{k} \lambda_{k} L_{k}(t)
$$

\footnotetext{
2 Depending on the actual choice of $\beta^{[k]}$ such functions might either have an analytical expression or need to be determined by numerical integration. Also, their partial derivatives with respect to $V_{0}$ and $\alpha$ will be assumed to be known in later results, again by symbolic or numeric computation.
} 
Also, no trajectory in $\mathcal{G}$ can produce $\nu(t)$ rendering $\eta(t)<0$ as $V$ is non-negative. Now, as $0 \leq \eta(t) \leq \psi_{\max }$, with $\psi_{\max }$ finite by assumption, we can assert that $0 \leq t^{-1} \int_{0}^{t} \eta(\tau) d \tau \leq \psi_{\max }$ for $t>0$, and that liminf of the average $\eta$ exists due to $\eta$ being bounded. As $\eta=\alpha^{-1}(\nu-\dot{\eta})$, for $t>0$ we have

$$
\begin{gathered}
\frac{1}{t} \int_{0}^{t} \eta(\tau) d \tau=\sum_{k=1}^{S} \lambda_{k} \frac{1}{\alpha t} \int_{0}^{t} L_{k}(\tau) d \tau+\frac{1}{\alpha t}(\eta(t)-\eta(0)) \\
\leq \alpha^{-1} \sum_{k=1}^{S} \lambda_{k} \bar{\beta}^{[k]}(t, t)+\frac{1}{\alpha t}(\eta(t)-\eta(0))
\end{gathered}
$$

As the required limits exist we can prove that

$$
\liminf _{t \rightarrow \infty} \frac{1}{t} \int_{0}^{t} \eta(t) d t \leq \alpha^{-1} \sum_{k} \lambda_{k} \bar{\beta}_{\infty}^{[k]}
$$

thus, for any $\varepsilon>0$ there exists $t>0$ such that $\frac{1}{t} \int_{0}^{t} \eta(t) d t \leq \alpha^{-1} \sum_{k} \lambda_{k} \bar{\beta}_{\infty}^{[k]}+\varepsilon$. Now, the mean-value theorem for integrals ensures that there exists $t_{1} \leq t$ achieving a value $\eta\left(t_{1}\right)$ lower or equal than its average in $[0, t]$. So, $\Theta_{v}$ will be entered at such time $t_{1}$.

With the above results, the state is guaranteed to never leave $\Theta_{T}$, and ultimately lie and not escape from $\Theta_{\infty}$, entering, too, $\Theta_{v}$ at least once with controller $u(x)$. Clearly, any $\Theta(\tau)$ in Theorem 1 is a guaranteed-visit set from $\Theta(0)$; however, $\Theta_{v}$ is independent of $V_{0}$. This will be useful in next section, being $V_{0}$ a decision variable there.

Tight constraints. $\quad \beta^{[k]}$ might be arbitrary, but some choices of them are unnecessarily conservative. Conservatism can be reduced via the lemma below:

Lemma 2 Given an arbitrary $\beta^{[k]}(t, h)$, there exists $\beta^{*}(t, h) \leq \beta^{[k]}(t, h)$ for which $\int_{0}^{h} L_{k}(t-\tau) d \tau \leq \beta^{*}(t, h)$ and

$$
\beta^{*}(t, h)=\min \left(\beta^{[k]}(t, h), \inf _{0<\xi<h}\left(\beta^{*}(t, \xi)+\beta^{*}(t-\xi, h-\xi)\right)\right)
$$

Proof: For any $0<\xi<h$ :

$$
\begin{gathered}
\int_{0}^{h} L_{k}(t-\tau) d \tau=\int_{0}^{\xi} L_{k}(t-\tau) d \tau+\int_{\xi}^{h} L_{k}(t-\tau) d \tau= \\
\int_{0}^{\xi} L_{k}(t-\tau) d \tau+\int_{0}^{h-\xi} L_{k}(t-\xi-\tau) d \tau
\end{gathered}
$$

So, we can assert

$\int_{0}^{h} L_{k}(t-\tau) d \tau \leq \inf _{0<\xi<h}\left(\beta^{[k]}(t, \xi)+\beta^{[k]}(t-\xi, h-\xi)\right)$

Hence, we can bound the integral in interval $[0, h]$ from previously-obtained bounds for intervals $[0, \xi]$, and $[0, h-\xi]$, being $\xi$ arbitrary. Given this bound, it is conservative to use a function where the assertion below does not hold for all $t, h$ :

$$
\beta^{[k]}(t, h) \leq \inf _{0<\xi<h}\left(\beta^{[k]}(t, \xi)+\beta^{[k]}(t-\xi, h-\xi)\right)
$$

Indeed, in such a case, generating a new function

$\beta_{1}^{*}(t, h)=\min \left(\beta^{[k]}(t, h), \inf _{0<\xi<h}\left(\beta^{[k]}(t, \xi)+\beta^{[k]}(t-\xi, h-\xi)\right)\right)$

would reduce conservatism if $\beta_{1}^{*}$ were used instead of $\beta^{[k]}$. Obviously $\beta_{1}^{*}$ could be plugged in at the right-hand side and the process repeated, obtaining $\beta_{2}^{*}, \beta_{3}^{*}, \ldots$ until convergence, letting $\beta^{*}:=\beta_{\infty}^{*}$. The recursion would give rise to a final $\beta^{*}$ verifying (15).

\section{Polynomial analysis and controller design}

In some cases, conditions in Theorem 1 can be handled via SOS software, if a polynomial $V(x)$ is sought and $\dot{x}$ in (1) is polynomial or convex combination thereof, obtained with $[6,17]$ from non-polynomial $f, B$ and $e$ :

$$
\begin{array}{r}
f(x):=\sum_{i=1}^{r} \mu_{i}(x) f_{i}(x), \quad B(x):=\sum_{i=1}^{r} \mu_{i}(x) B_{i}(x), \\
e(x, w):=\sum_{i=1}^{r} \mu_{i}(x) e_{i}(x, w)
\end{array}
$$

with $\mu_{i} \geq 0, \sum \mu_{i}=1$. A polynomial $u(x)$ may also be proposed, or extended to $u(x):=\sum_{i=1}^{r} \mu_{i}(x) u_{i}(x)$. A component-wise input constraint will be also considered:

$$
\left|u^{[j]}(x)\right| \leq \varsigma_{j} \quad \forall x \in \Omega
$$

where $u^{[j]}$ denotes the $j$-th element of the input vector.

Given two semialgebraic sets $R, Q$, notation $\mathcal{P} \mathcal{S}[R \subset Q]$ will denote any sufficient condition for set $R$ being a subset of $Q$, such as the Positivstellensatz ones in [21], implementations in [12], etc. These well-known conditions involve some multipliers as additional decision variables ${ }^{3}$. Let us denote as $\mathcal{T}:=\{(x, w): x \in \Omega, w \in \mathcal{W}(x)\}$.

Theorem 2 Given user-defined semialgebraic sets $\Omega$, $\Theta_{i}, \Theta_{V}$ and $\Theta_{f}$, being $\Omega$ an open set, if there exists a polynomial function $V(x)$, a level value $V_{0}$, scalars $\varepsilon_{1}>0$, $\varepsilon_{2}>0, \alpha>0, \lambda_{k} \geq 0$, a controller $u(x)$ and Positivstellensatz multipliers fulfilling conditions:

$$
\begin{aligned}
\mathcal{P S} & {\left[\mathcal { T } \subset \left\{(x, w): \frac{\partial V}{\partial x}(f(x)+B(x) u(x)\right.\right.} \\
& \left.\left.+e(x, w))+\alpha \cdot V-\sum_{k=1}^{S} \lambda_{k} L_{k}(w, x) \leq 0\right\}\right]
\end{aligned}
$$

\footnotetext{
3 For instance, a particular choice for $\mathcal{P S}[R \subset Q]$ with $R=$ $\{r(x) \geq 0\}$ and $Q=\{q(x)>0\}$ could be the existence of $v>$ 0 and polynomial multipliers $\tau_{1}(x), \tau_{2}(x)$ such that $\tau_{1}(x)$, $\tau_{2}(x)$ and $\tau_{1}(x) r(x)-\tau_{2}(x)(q(x)-v)$ are SOS polynomials (the quadratic case ends up in the S-procedure); actually $R \subset\{q(x) \geq v\}$. The reader is referred to the cited works for software and implementation details.
} 


$$
\begin{gathered}
\mathcal{P S}\left[\Theta_{i} \subset\left\{x: V(x) \leq V_{0}\right\}\right] \\
\mathcal{P S}\left[\left\{x: V(x) \leq \psi_{\max }\left(V_{0}, \alpha, \lambda\right)\right\} \subset \Omega\right] \\
\mathcal{P S}\left[\left\{x: V(x) \leq \psi_{\infty}(\alpha, \lambda)+\varepsilon_{1}\right\} \subset \Theta_{f}\right] \\
\mathcal{P S}\left[\left\{x: V(x) \leq \psi_{v}(\alpha, \lambda)+\varepsilon_{2}\right\} \subset \Theta_{V}\right] \\
\mathcal{P S}\left[\left\{x: V \leq \psi_{\max }\left(V_{0}, \alpha, \lambda\right)\right\} \subset\right. \\
\left.\left\{x: \varsigma_{j}^{2}-\left(u^{[j]}(x)\right)^{2} \geq 0\right\}\right] \quad \forall j
\end{gathered}
$$

Then $\Omega$ is robustly inescapable and $\Theta_{f}$ is ultimately inescapable from $\Theta_{i}$ with $u(x)$, fulfilling the bound (19) for any trajectory starting in $\left\{x: V(x) \leq V_{0}\right\}$ which, by (21), contains $\Theta_{i}$. Also $\Theta_{V}$ is a guaranteed-visit set.

Proof: Expression (20) is a sufficient condition for (7), and so they are (21) and (22) for (9). Then, (23) forces the ultimately inescapable set $\Theta_{\infty}$ to be inside the prefixed $\Theta_{f}$, and (25) ensures (19) on $\Theta_{T}$. Last, (24) ensures $\Theta_{v} \subset \Theta_{V}$.

In case $\psi_{\infty}=\psi_{\max }$, from Corollary $1(\mathrm{a}), \varepsilon_{1}$ can be set to 0 . Also, in the case $\alpha=0,(23)$ and (24) should be removed. Convex sums in both (18) and the control law will, too, need sufficient copositive sum relaxations of (20), such as Polya [19, Sec. 7.1].

Optimisation. If one set (either $\Theta_{i}, \Theta_{f}, \Theta_{V}$ or $\Omega$ ) is replaced by a parameterised prefixed-shape set $H_{\sigma}=$ $\left\{x: \max _{k=1, \ldots, k_{M}} h_{k}(x) \leq \sigma\right\}$, leaving the other two sets fixed, Theorem 2 can be reformulated as an optimisation problem. Then, size parameter $\sigma$ or some performance/ disturbance bounds can be suitably optimised in order to solve problems such as:

P1: Smallest inescapable set from given initial conditions. Given an initial condition set $\Theta_{i}$, set $\Omega=$ $\operatorname{int}\left(H_{\sigma}\right)$ and minimise the size parameter $\sigma$.

P2: Largest admissible initial condition set. Given $\Omega$, maximise $\sigma$ such that $H_{\sigma} \subset\left\{V \leq V_{0}\right\}$.

P3: Smallest ultimately inescapable set from given initial conditions. Given an initial condition set $\Theta_{i}$, minimise $\sigma$ such that $\Theta_{\infty} \subset H_{\sigma}$.

P4: Smallest guaranteed-visit set from given initial conditions. Given an initial condition set $\Theta_{i}$, minimise $\sigma$ such that $\Theta_{v} \subset H_{\sigma}$.

P5: Closed-loop performance goal. Optimising some classic (quadratic cost bound, norm bound, etc.) performance criteria subject to some of the above inescapability conditions.

P6: Disturbance bounds. Optimising parameters in $\beta^{[k]}$ subject to particular inescapability conditions.

Numerically, conditions in Theorem 2 are nonconvex ${ }^{4}$ because of the product $\alpha V$ and $\frac{\partial V}{\partial x} B(x) u(x)$, and the dif-

\footnotetext{
${ }^{4}$ Only in some very particular cases of bound $\beta$ and linear polytopic systems, quadratic $V(x)$, Theorem 2 reduces to cases in literature in the introduction, some of them convex.
}

ferent Positivstellensatz multipliers which may multiply $V(x), u(x)$ or the parameters $V_{0}, \psi_{\max }, \psi_{\infty}$, etc. Also, $\psi_{\max }\left(V_{0}, \alpha, \lambda\right)$ and $\psi_{\infty}(\alpha, \lambda)$ are nonlinear in their arguments (perhaps needing approximate numerical evaluation). In order to handle nonlinearity in decision variables with SDP software, a gradient-based iterative linearisation [13], possibly converging to a local optimum, will be pursued, as follows:

Algorithm. Consider some decision variables $Q$ and a generic problem of minimising $f(Q)$, subject to a polynomial $g(Q, x)$ being SOS, denoted as $S O S(g(Q, x))$. Here, $f$ is maybe non-convex in $Q$. The algorithm below will succeed in finding a local minimum ( $\nabla$ denotes gradient with respect to $Q$ ):

(1) Start with $k=0$, and a feasible solution $Q_{k}:=Q_{0}$. Such solution, problem dependent, might involve particular cases in literature, constraint softening in initial iterations, etc., see example below.

(2) Minimise the linearised $f\left(Q_{k}\right)+\nabla f\left(Q_{k}\right) \cdot \delta Q$, subject to $S O S\left(g\left(Q_{k}, x\right)+\nabla g\left(Q_{k}, x\right) \cdot \delta Q\right)$. Note that this is a convex SOS problem on the new decision variables $\delta Q$. Denote as $\delta Q^{*}$ the optimal solution.

(3) Starting with $j=0$, define $\lambda:=2^{-j}$, and increase $j$ until ${ }^{5} Q_{k+1}=Q_{k}+\lambda \delta Q^{*}$ fulfills $f\left(Q_{k+1}\right)<f\left(Q_{k}\right)$ and $S O S\left(Q_{k+1}, x\right)$ or $j=j_{M A X}$.

(4) If $j=j_{M A X}$, then STOP (a local minimum has been reached if step $\lambda$ gets negligible). ELSE, set $k=k+1$ and go to step 2 .

\section{Example}

Let us consider the following nonlinear system:

$\dot{x}=\left(\begin{array}{cc}-3 & 0.5 \\ 0 & -4.1+3.8 \sin \left(x_{1}\right)\end{array}\right) x+\left(\begin{array}{l}0 \\ 1\end{array}\right) u+\left(\begin{array}{c}0.9 \\ -0.56\end{array}\right) w$

in a modelling region $\Omega$ defined as an open circle with radius $R$. The control action bound in (19) has been set to $\varsigma=1$. Note that the origin is an equilibrium point.

A single disturbance bound (2) with $L(w, x)=w^{2}$ has been considered, with $\bar{\beta}(h)^{1 / 2}$ (not depending on $t$ ) depicted in Figure 1: disturbances with a high RMS value in short time are allowed; however, the allowed RMS average is a decreasing function of $h$ (up to an infinite-time average $\left.\bar{\beta}_{\infty}=0.1\right)$. Figure 1 has been obtained with $\beta(h)=0.1 h+0.5 e^{-h}+0.4 h \cdot e^{-0.1 h}$.

The non-linearity $\sin \left(x_{1}\right)$, modelled via a Taylor series approach (degree 3 ), can be expressed as

$$
\sin \left(x_{1}\right)=\mu_{1}\left(x_{1}\right)\left(x_{1}-x_{1}^{3} / 6\right)+\mu_{2}\left(x_{1}\right)\left(x_{1}+g(R) x_{1}^{3}\right)
$$

\footnotetext{
${ }^{5}$ Note that, if $\nabla f\left(Q_{k}\right) \neq 0$, there exists a small enough $\lambda>0$ such that $f\left(Q_{k}+\lambda \delta Q^{*}\right)<f\left(Q_{k}\right)$, so if the gradient points inwards of the feasible set, some $j$ will exist for sure.
} 


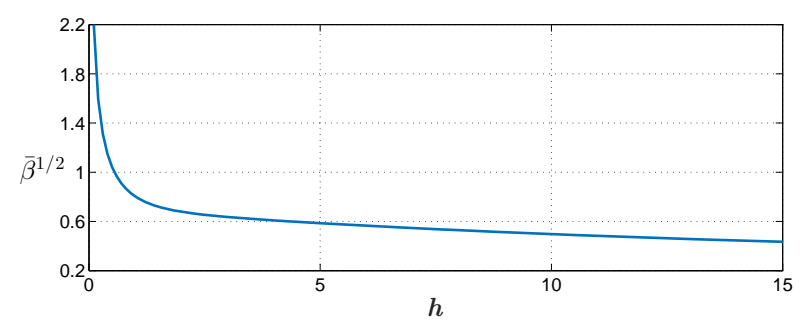

Fig. 1. Chosen RMS bound function $\bar{\beta}^{1 / 2}$ in an interval $h$.

being $g(R)=(\sin (R)-R) / R^{3}$ for $R>0$,

$$
\mu_{1}\left(x_{1}\right)=\frac{\sin \left(x_{1}\right)-\left(x_{1}+g(R) x_{1}^{3}\right)}{(-g(R)-1 / 6) x_{1}^{3}}
$$

and $\mu_{2}=1-\mu_{1}$. Replacing (27) in (26), we get a representation (18). Then, with such model, some examples of application of Theorem 2 will be now shown. Multipliers and implementation details are omitted for brevity.

Case 1: Inescapable set from origin. By Corollary 1(b), transient and ultimate inescapable sets are identical in this particular case, i.e., $\Theta_{\infty}=\Theta_{T}$, as we set $V_{0}=0$. The objective is minimising the radius $\sqrt{\sigma}$ of a circular region $\Theta_{f}=H_{\sigma}:=\left\{x^{T} x \leq \sigma\right\}$, problem P3 (equivalent to P1). Quadratic and linear structures have been arbitrarily chosen for $V(x)$ and $u(x)$, respectively.

In order to obtain such $\sigma$, the initial $\Omega$ is set as a small "seed" circle around the origin. Then, feasible decision variable values in Theorem 2 are obtained without considering constraint (22), solving problem P3 for the smallest circular $\Theta_{f}$. Subsequently, if $\Theta_{f} \not \subset \Omega, \Omega$ is enlarged so that it is slightly larger than $\Theta_{f}$, and computations are repeated until such situation does not arise (details omitted for brevity).

As a result of the above, setting $\Theta_{f}$ to be the circle of radius 0.269 and $\Omega$ an open circle of radius $R=0.27$ results in a feasible problem for Theorem 2 . The linear controller $u(x)=2.9019 x_{1}-3.7984 x_{2}$ can be used to prove an inescapable set starting from equilibrium $\left(\Theta_{i}=\right.$ $0)$ given by the small purple ellipsoid $\Theta_{T}^{\left[\Theta_{i}=0\right]}$ in Figure 2. The set $\Theta_{f}$ is the dotted black circle labelled as $R_{1}$.

Case 2: computation of largest initial condition set for a given inescapable region $\Omega$ (problem P2). Let us set $\Omega$ as the open circle of radius $\mathrm{R}=3$, and a degree 4 choice for polynomial $V(x)$. A polynomial controller $u=$ $\sum_{i=1}^{2} \mu_{i}(x) u_{i}(x)$ with gains $(28),(29)$ below, proves that initial conditions in the blue $\Theta_{i}$ set in Figure 2 make $\Omega$ (outer gray circle) inescapable from them (indeed $\Theta_{T}$ is almost tangent to the boundary of $\Omega$, see the brown curve; the value of $\nu$ in footnote 3 was $10^{-3}$ ). The set has been obtained in an iterative maximisation of the minimum radius $\sqrt{\sigma}$ of $\Theta_{i}:=H_{\sigma}$, resulting in $\sqrt{\sigma}=2.56$ (dotted red circle labelled as $R_{2}$ ). Finally, the green curve in Figure 2 depicts the boundary of the guaranteed-visit set $\Theta_{v}$ with the obtained $V(x), \alpha, \lambda$ and $\epsilon=10^{-3}$ by Lemma 1.

$$
\begin{gathered}
u_{1}(x)=-0.065691 x_{1}^{3}+0.016334 x_{1}^{2} x_{2}-0.036757 x_{1} x_{2}^{2} \\
-0.0014564 x_{1}^{2}-0.030947 x_{1} x_{2}+0.51542 x_{1} \\
+0.016334 x_{1}^{2} x_{2}-0.036757 x_{1} x_{2}^{2}+0.0084773 x_{2}^{3} \\
\quad-0.030947 x_{1} x_{2}-0.011761 x_{2}^{2}-0.23688 x_{2} \\
u_{2}(x)=-0.043735 x_{1}^{3}+0.0058491 x_{1}^{2} x_{2}-0.014259 x_{1} x_{2}^{2} \\
+0.002483 x_{1}^{2}-0.063374 x_{1} x_{2}+0.3396 x_{1} \\
+0.0058491 x_{1}^{2} x_{2}-0.014259 x_{1} x_{2}^{2}-0.0090187 x_{2}^{3} \\
\quad-0.063374 x_{1} x_{2}-0.011354 x_{2}^{2}-0.15678 x_{2}
\end{gathered}
$$

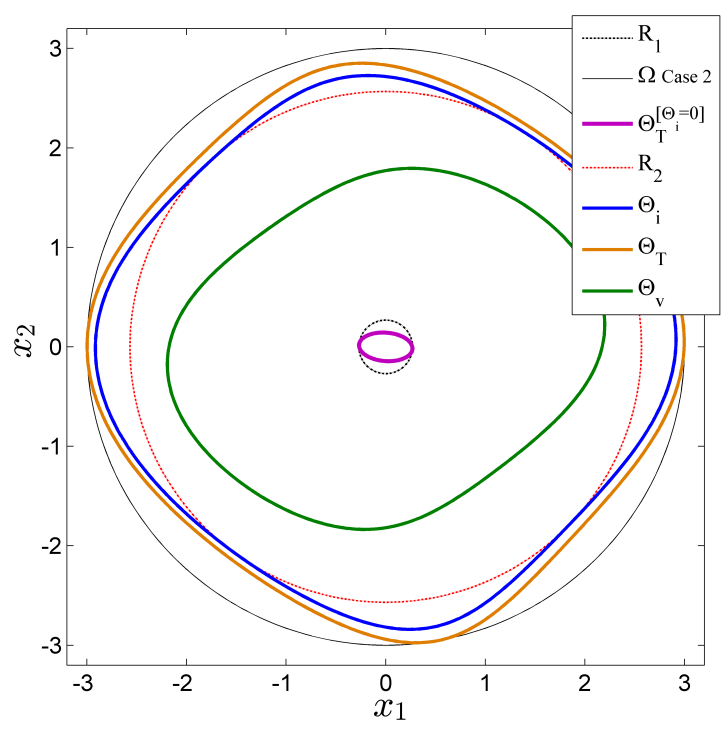

Fig. 2. Inescapable, guaranteed-visit and initial sets for Case 1 (purple, dashed black) and Case 2 (rest of lines).

\section{Conclusions}

This paper identifies four interrelated sets in the dynamic behaviour of a nonlinear system subject to nonvanishing disturbances, constrained by finite-interval integral bounds. The relevant sets are: 1) the initial condition set, 2) inescapable (at any time) sets, 3) ultimately inescapable sets, when $t$ is large and 4) guaranteed-visit sets. Level sets of a Lyapunov-like function constrain the shape of such sets.

Inescapable sets are needed in proving controller validity when models are local. Also, the guaranteed-visit set $\Theta_{v}$ gives the opportunity of "capturing" the state near the origin and changing the control law to another with better provable performance (left for further research).

When the involved models are polynomials (or convex combinations thereof) some sufficient sum-of-squares conditions can be set up in order to solve some optimisation problems on the size of one of the sets given bounds for the remaining ones. In many cases, such conditions are nonconvex so iterative procedures are needed. 


\section{Acknowledgements}

The research leading to these results has received funding from the European Union (FP7/2007-2013 $\mathrm{n}^{\circ} 604068$ ) and from the Spanish Government (MINECO/ FEDER DPI2015-70975-P, DPI2011-27845-C02-01).

\section{References}

[1] J. Abedor, K. Nagpal, and K. Poolla. A linear matrix inequality approach to peak-to-peak gain minimization. Int. J. of Robust and Nonlinear Control, 6(9-10):899-927, 1996.

[2] C. P. Bechlioulis and G. A. Rovithakis. Adaptive control with guaranteed transient and steady state tracking error bounds for strict feedback systems. Automatica, 45(2):532-538, 2009.

[3] F. Blanchini. Set invariance in control. Automatica, 35(11):1747 - 1767, 1999.

[4] F. Blanchini and M. Sznaier. Persistent disturbance rejection via static-state feedback. Automatic Control, IEEE Transactions on, 40(6):1127-1131, Jun 1995.

[5] Y.Y. Cao, Z. Lin, and Y. Shamash. Set invariance analysis and gain-scheduling control for LPV systems subject to actuator saturation. Systems \& Control Letters, 46(2):137 151, 2002.

[6] G. Chesi. Estimating the domain of attraction for nonpolynomial systems via LMI optimizations. Automatica, 45(6):1536 - 1541, 2009.

[7] G. Chesi. Domain of Attraction Analysis and Control Via SOS Programming. Springer Verlag, 2011.

[8] P. Gahinet and P. Apkarian. A linear matrix inequality approach to $H_{\infty}$ control. Inter. Journal of Robust and Nonlinear Control, 4:421-448, 1994.

[9] H. Haimovich and M. M. Seron. Bounds and invariant sets for a class of switching systems with delayed-state-dependent perturbations. Automatica, 49(3):748-754, 2013.

[10] E. J. Hancock and A. Papachristodoulou. Generalised absolute stability and sum of squares. Automatica, feb 2013.

[11] T. Hu, Z. Lin, and B. M. Chen. An analysis and design method for linear systems subject to actuator saturation and disturbance. Automatica, 38(2):351 - 359, 2002.

[12] Z. Jarvis-Wloszek, R. Feeley, W. Tan, K. Sun, and A. Packard. Control applications of sum of squares programming. In D. Henrion and A. Garulli, editors, Positive Polynomials in Control, pages 3-22. Springer, 2005.

[13] S. Kanev, C. Scherer, M. Verhaegen, and B. De Schutter. Robust output-feedback controller design via local BMI optimization. Automatica, 40(7):1115 - 1127, 2004.

[14] E. Kofman, J. A. De Doná, and M. M. Seron. Probabilistic set invariance and ultimate boundedness. Automatica, 48(10):2670-2676, 2012.

[15] R. M. Palhares and Pedro L.D. Peres. Robust filtering with guaranteed energy-to-peak performance an LMI approach. Automatica, 36(6):851 - 858, 2000.

[16] J. L. Pitarch, A. Sala, and C. V. Ariño. Closed-form estimates of the domain of attraction for nonlinear systems via fuzzy polynomial models. IEEE Trans. on Cybernetics., 44(4):526538, Apr 2014.

[17] A. Sala and C. Ariño. Polynomial fuzzy models for nonlinear control: A Taylor series approach. Fuzzy Systems, IEEE Transactions on, 17:1284-1295, aug 2009.
[18] J. V. Salcedo, M. Martínez, and S. García-Nieto. Stabilization conditions of fuzzy systems under persistent perturbations and their application in nonlinear systems. Eng. Appl. Artificial Intelligence, 21(8):1264 - 1276, 2008.

[19] C. W. Scherer. LMI relaxations in robust control. European Journal of Control, 12(1):3-29, 2006.

[20] J. S. Shamma. Analysis and design of gain scheduled control systems. $\mathrm{PhD}$ thesis, Dept. of Mechanical Engineering. Massachusetts Institute of Technology. USA, 1988.

[21] G. Stengle. A Nullstellensatz and a Positivstellensatz in semialgebraic geometry. Mathematische Annalen, 207(2):8797, 1974. 